\title{
DESENVOLVENDO COMPETÊNCIAS NA APRENDIZAGEM DE ESTUDANTES DE MEDICINA VETERINÁRIA
}

\author{
Cláudia Turra Pimpão ${ }^{1}$ \\ Dariane Cristina Catapan ${ }^{2}$ \\ Rita Maria Venâncio Mangrich Rocha ${ }^{3}$ \\ Kung Darh $\mathrm{Chi}^{3}$ \\ Valeria Natasha Teixeira ${ }^{4}$ \\ Humberto Maciel França Madeira ${ }^{1}$ \\ Ana Lúcia Michelotto ${ }^{5}$ \\ Mirian Célia Castellain Guebert ${ }^{1}$ \\ Javier Burchard ${ }^{6}$
}

\begin{abstract}
RESUMO
Atualmente para os professores universitários obterem a atenção do estudante em sala de aula, é necessário romper alguns paradigmas e ser inovador na forma de ensinar para poder manterse motivado, porque concorrer com a facilidade de acesso as informações juntamente com o mundo tecnológico a disposição dos alunos, torna-se um desafio para toda a comunidade acadêmica. A educação universitária necessita de implementação de novos modos de se pensar e praticar o processo de ensino e aprendizagem. A metodologia de ensino deve ser ativa e inovadora, levando em conta os saberes prévios dos alunos, sua realidade social, política, profissional e cultural. As metodologias de projetos que estimulem a pesquisa, resultam em descobertas significativas e aplicáveis à realidade de cada discente possibilitando ao docente ser mediador do processo de ensino e aprendizagem, criando cenários que rompam com a visão tradicional de ver e compreender as coisas por parte dos discentes. O objetivo do presente estudo é eleger as possíveis competências para serem utilizadas como metodologia de ensino e aprendizagem de estudantes de bacharelado em medicina veterinária na PUCPR. As competências desenvolvidas no Curso de Medicina Veterinária da PUCPR são pautadas em desenvolver competências basilares e específicas e são as mínimas competências esperadas que médicos veterinários recém-formados possuam, a fim de garantir serviços veterinários de alta qualidade.
\end{abstract}

Palavras-chave: Metodologias ativas, educação superior, diretrizes curriculares, ensinoaprendizagem.

\section{DEVELOPING SKILLS IN LEARNING OF VETERINARY MEDICINE STUDENTS}

\begin{abstract}
Currently for academics get the student's attention in the classroom, it is necessary to break some paradigms and be innovative in the way of teaching in order to remain motivated because compete with the ease of access to information along with the technological world layout students, it becomes a challenge for the entire academic community. University

\footnotetext{
${ }^{1}$ Professora titular da Pontifícia Universidade Católica do Paraná. Correspondência.

${ }^{2}$ Doutoranda em Saúde, Tecnologia e Produção Animal Integrada pela Pontifícia Universidade Católica do Paraná - PUCPR

${ }^{3}$ Professora Adjunto III da Pontifícia Universidade Católica do Paraná.

${ }^{4}$ Professora do Curso de Medicina Veterinária da Pontifícia Universidade Católica do Paraná.

${ }^{5}$ Escola de Saúde, Agrociências e Biotecnologia, Pontifícia Universidade Católica do Paraná.

${ }^{6}$ Médico Veterinário, Pontifícia Universidade Católica do Paraná.
} 
education needs to implement new ways of thinking and practicing the teaching and learning process. The teaching methodology should be active and innovative, taking into account the previous knowledge of the students, their social, political, professional and cultural reality. The project methodologies that encourage research, result in significant and relevant findings to the reality of each student enabling the teacher to be a mediator in the process of teaching and learning, creating scenarios that break with the traditional view of seeing and understanding things by the students. The aim of this study is to elect the possible skills to be used as a teaching and learning methodology of bachelor's students in veterinary medicine in PUCPR. The skills developed in the course of Veterinary Medicine at PUCPR are guided in developing basic and specific skills and are the minimum skills expected that newly graduated veterinarians have in order to ensure high quality veterinary services.

Keywords: Active methodologies, higher education, curriculum guidelines, teaching and learning.

\section{DESARROLLO DE COMPETENCIAS EN APRENDIZAJE DE LOS ESTUDIANTES EN MEDICINA VETERINARIA}

\section{RESUMEN}

Actualmente para que el professor académico llamen la atención del estudiante en el aula, es necesario romper algunos paradigmas y ser innovadores en la forma de enseñar con el fin de mantener la motivación porque competir con la facilidad de acceso a la información, junto con el diseño mundo tecnológico estudiantes, se convierte en un desafío para toda la comunidad académica. Una educación universitaria requiere la implementación de nuevas formas de pensar y practicar el proceso de enseñanza y aprendizaje. La metodología de enseñanza debe ser activa e innovadora, teniendo en cuenta los conocimientos previos de los estudiantes, su cultura social, política, profesional y. Las metodologías de proyectos que fomenten la investigación, dar lugar a conclusiones significativas y relevantes a la realidad de cada estudiante permitiendo a los profesores para mediar en el proceso de enseñanza y aprendizaje, la creación de escenarios que rompen con la visión tradicional de ver y entender las cosas por los estudiantes. El objetivo de este estudio es para elegir las posibles habilidades que se puedan utilizar como una metodología de enseñanza y el aprendizaje de los estudiantes de licenciatura en medicina veterinaria en la PUCPR. Las habilidades desarrolladas en el curso de Medicina Veterinaria PUCPR se guían en el desarrollo de habilidades básicas y específicas y son las competencias mínimas que se espera que los veterinarios recién graduados tienen el fin de garantizar los servicios veterinarios de alta calidad.

Palabras clave: Metodologías activas, la educación superior, lineamientos curriculares, la enseñanza y el aprendizaje.

\section{INTRODUÇÃO}

Atualmente, para os professores universitários manterem a atenção do estudante em sala de aula, é necessário romper alguns paradigmas, ou seja, ser inovador na forma de ensinar para poder manter a motivação, pois concorrer com a facilidade de acesso as informações juntamente com o mundo tecnológico a disposição dos alunos, torna-se um desafio para toda a comunidade acadêmica. 
Isso é um desafio, pois as atuais diretrizes curriculares nacionais - DCN, da educação universitária apontam para a necessidade de implementação de novos modos de se pensar e praticar o processo de ensino-aprendizagem $(1,2)$. As DCNs preveem mudanças curriculares no processo de ensino-aprendizagem que orientem uma formação profissional, dando protagonismo aos discentes $(3,4,5)$.

Esse processo ensino-aprendizagem requer uma metodologia de ensino ativa e inovadora, levando em conta os saberes prévios dos alunos, sua realidade social, política, profissional e cultural. As metodologias de projetos que estimulam a pesquisa resultam em descobertas significativas e aplicáveis à realidade de cada discente. Tal metodologia possibilita ao docente ser mediador do processo de ensino e aprendizagem, criando cenários que rompam com a visão tradicional de ver e compreender as coisas por parte dos discentes (6).

Para que este modelo do processo ensino-aprendizagem seja eficiente, são necessárias mudanças no perfil docente e discente, e isso exige uma transformação na formação superior, do atual modelo baseado na transmissão de conhecimentos e na memorização para um modelo educativo embasado na formação por competências, que é a aquisição de conteúdos para que o aluno consiga adquirir habilidades, conhecimentos e atitudes centradas no processo de aprendizagem (7).

A aprendizagem por competência pode ser considerada como uma metodologia inovadora e norteia-se pela ação principal sendo do aluno e não do professor, portanto o professor atua como um facilitador ou mediador. Desta forma, aprender por competência significa uma abordagem didática orientada para a execução imediata de habilidades, sendo um enfoque que contempla uma aprendizagem necessária para que o estudante atue de maneira ativa, responsável e criativa, com habilidades de comunicação oral e escrita, habilidades de avaliação crítica e habilidades de caráter pessoal, para a construção de seu projeto de vida, tanto pessoal e social como profissional (8).

Atualmente os docentes de educação superior devem ensinar seus estudantes a aprender e também a tomar iniciativas, ao invés de serem somente fontes de conhecimento (9).

O contexto da profissão do Médico Veterinário é amplo, pois o perfil do profissional a ser formado é generalista e serão profissionais responsáveis pela promoção da saúde e do bem-estar animal, pela saúde pública e segurança alimentar, portanto trabalha para a humanidade (4). Considerando o exposto acima, aliado aos princípios institucionais são a base para a proposta educativa e orienta a ação pedagógica do Curso de Medicina Veterinária na Pontifícia Universidade Católica do Paraná (PUCPR) que acredita que o conhecimento humano e a busca da verdade dão-se na mútua valorização da ciência, fé e razão, calcada na ética e em valores cristãos; de vivência conforme os valores maristas, corporificando a sua responsabilidade de formação integral do profissional $(10,11,12,13)$.

O objetivo do presente estudo é eleger as possíveis competências para serem utilizadas como metodologia de ensino e aprendizagem de estudantes de bacharelado em medicina veterinária na PUCPR, bem como comparar este projeto pedagógico com as recomendações da Organização Mundial para a Saúde Animal (OIE) para educação em Medicina Veterinária.

\section{MATERIAL E MÉTODOS}

Este trabalho caracteriza-se, quanto aos objetivos, como uma pesquisa descritiva, pois têm como finalidade principal a descrição das características de determinada população ou fenômeno (14). A pesquisa descritiva apenas captura, demonstra e faz o registro escrito de um cenário de determinada situação (15). Em relação aos procedimentos, o presente trabalho utiliza a pesquisa bibliográfica, definida como o levantamento de referências teóricas já 
analisadas, e publicadas por meios escritos e eletrônicos, como livros, artigos científicos, páginas de web sites (16).

\section{RESULTADOS E DISCUSSÃO Metodologia por competência}

Competência refere-se à capacidade de mobilizar múltiplos recursos, entre os quais os conhecimentos teóricos e experiências da vida pessoal e profissional, para responder às diferentes demandas das situações de trabalho. Apoia-se, portanto, no domínio de saberes, mas não apenas de saberes teóricos, e refere-se à atuação em situações complexas (17).

A competência é uma palavra do senso comum, utilizada para designar uma pessoa qualificada para realizar alguma coisa. O seu oposto, não implica apenas a negação desta capacidade, mas guarda um sentimento depreciativo. O conceito de competência é definido como uma combinação de conhecimentos (adquiridos pelas informações e conteúdos), habilidades e atitudes (18).

O conhecimento se refere ao saber o que fazer; a habilidade está relacionada ao saber como fazer; e a atitude, ao querer fazer. A noção de competência explicita a necessidade da experiência prática e, por vezes, específica, para que o desempenho esperado seja alcançado (19).

A educação ao longo da vida se baseia nos "Quatro Pilares da Educação" propostos pela Unesco no documento "Educação: Um Tesouro a Descobrir" (20): aprender a conhecer, aprender a fazer, aprender a conviver e aprender a ser. Para que sejam possíveis as reformas educacionais, seja na elaboração dos programas ou na definição de novas políticas pedagógicas, as instituições de ensino devem adotar em sua totalidade os quatro pilares da educação (Quadro 1) como uma nova proposta ensino-aprendizagem, objetivando desenvolver as competências nos discentes.

$\mathrm{O}$ que diferencia os processos de aprendizagem que um indivíduo experimenta no seu dia a dia do processo de aprendizagem que esse mesmo indivíduo vivencia em sala de aula, é a intencionalidade (22), pois a aprendizagem é um processo de mudança no comportamento, seja ela de natureza cognitiva, psicomotora ou afetiva, provocado por uma experiência (19).

No processo de ensino-aprendizagem existe uma intencionalidade: produzir aprendizagem. No ensino, todas as atividades são concebidas e planejadas com o objetivo de produzir uma aprendizagem nos seus ouvintes. Por isso, as informações deverão ser organizadas e sistematizadas de forma a alcançar esse objetivo. Ao realizar esses procedimentos, o professor estará definindo uma estratégia de ensino-aprendizagem (22).

De acordo com a publicação "Estratégias de ensino-aprendizagem para desenvolvimento das competências humanísticas", elaborada pelo Conselho Federal de Medicina Veterinária (19), o processo de ensino-aprendizagem, para que seja efetivo, compreende diferentes fases que precisam, necessariamente, estar coordenadas entre si. Essas fases ou etapas podem ser divididas em: planejamento; execução de atividades de ensinoaprendizagem e avaliação das competências.

\section{Competências da medicina veterinária}

Para a OIE (23), os Médicos Veterinários, independentemente da sua área de prática profissional, são responsáveis pela promoção da saúde e do bem-estar animal, pela saúde pública e segurança alimentar, sendo os serviços veterinários considerados um bem público mundial. A educação veterinária é uma pedra basilar para assegurar que o Médico Veterinário receba uma formação que lhe garanta competências condizentes com o que se espera de sua atuação. 
E as competências específicas mencionadas pela OIE (24) são: epidemiologia, doenças de animais transfronteiriças, zoonoses (incluindo as doenças de origem alimentar), doenças emergentes e re-emergentes, programas de prevenção e controle de doenças, higiene de alimentos, produtos veterinários, bem-estar animal, legislação veterinária e ética, procedimentos gerais de certificação, e habilidades de comunicação.

Quadro 1. Pilares da educação e competências a serem desenvolvidas pelos discentes.

\begin{tabular}{|c|c|}
\hline NOME DO PILAR & DESCRIÇÃO DOS PILARES / COMPETÊNCIAS \\
\hline \multirow[b]{2}{*}{ Aprender a conhecer } & $\begin{array}{l}\text { Combinando uma cultura geral, suficientemente ampla, com a possibilidade de } \\
\text { estudar, em profundidade, um número reduzido de assuntos, ou seja: aprender a } \\
\text { aprender, para se beneficiar das oportunidades oferecidas pela educação ao longo da } \\
\text { vida (20). }\end{array}$ \\
\hline & $\begin{array}{l}\text { Assimilar conhecimentos científicos e culturais gerais e específicos, que se } \\
\text { completarão e atualização ao longo de toda a vida (5). } \\
\text { Possibilidade de aprendizagem e construção de conceitos relacionados aos temas, por } \\
\text { meio das problematizações, leituras, pesquisas, análises e mapeamento de situações- } \\
\text { problemas, contextualizadas ao campo de atuação (21). }\end{array}$ \\
\hline \multirow[b]{2}{*}{ Aprender a fazer } & $\begin{array}{l}\text { Adquirir não só uma qualificação profissional, mas, de uma maneira mais } \\
\text { abrangente, a competência que torna a pessoa apta a enfrentar numerosas situações e } \\
\text { a trabalhar em equipe (20). }\end{array}$ \\
\hline & $\begin{array}{l}\text { Adquirir procedimentos que ajudem a afrontar as dificuldades que se apresentem na } \\
\text { vida e na profissão (5). } \\
\text { Possibilidades de desenvolver e implementar a pesquisa, relatórios, sínteses e } \\
\text { projetos (21). }\end{array}$ \\
\hline \multirow{2}{*}{ Aprender a conviver } & $\begin{array}{l}\text { Desenvolvendo a compreensão do outro e a percepção das interdependências - } \\
\text { realizar projetos comuns e se preparar para gerenciar conflitos - no respeito pelos } \\
\text { valores do pluralismo, da compreensão mútua e da paz (20). }\end{array}$ \\
\hline & $\begin{array}{l}\text { Compreender melhor aos demais, o mundo e suas inter-relações (5). } \\
\text { Possibilidades de trabalhar em equipe, de desenvolver a tolerância, responsabilidade, } \\
\text { de comunicar-se e de produzir reflexões e ações coletivas }(21) \text {. }\end{array}$ \\
\hline \multirow[t]{2}{*}{ Aprender a ser } & $\begin{array}{l}\text { Desenvolver, o melhor possível, a personalidade e as condições de agir com uma } \\
\text { capacidade cada vez maior de autonomia, discernimento e responsabilidade pessoal. } \\
\text { Com essa finalidade, a educação deve levar em consideração todas as potencialidades } \\
\text { de cada indivíduo: memória, raciocínio, sentido estético, capacidades físicas, aptidão } \\
\text { para se comunicar (20). }\end{array}$ \\
\hline & $\begin{array}{l}\text { Atuar com autonomia, juízo, responsabilidade pessoal (5). } \\
\text { Possibilidade de autoconhecimento nas análises crítico-reflexivas do processo } \\
\text { ensino-aprendizagem, e nos processos desenvolvidos, durante a formação (21). }\end{array}$ \\
\hline
\end{tabular}

As competências avançadas listadas pela OIE (24) são: organização dos serviços veterinários, procedimentos de inspeção e certificação, gestão de doenças contagiosas, higiene de alimentos, aplicação de análises de risco, pesquisa, quadro do comércio internacional, administração e gestão.

Tanto as competências específicas quanto as avançadas, são as mínimas competências esperadas que médicos veterinários recém-formados possuam, a fim de garantir serviços veterinários de alta qualidade $(1,4,23)$.

\section{Diretrizes curriculares nacionais dos cursos de medicina veterinária}

A Resolução CNE/CES no 1/2003 estabelece que a formação do Médico Veterinário tem por objetivo dotar o profissional dos conhecimentos para desenvolver ações e resultados voltados à área de Ciências Agrárias no que se refere à Produção Animal, Produção de Alimentos, Saúde Animal e Proteção Ambiental, além das seguintes competências 
(gerais/humanísticas): atenção à saúde, tomada de decisões, comunicação, liderança, administração e gerenciamento, educação permanente (25).

Essas competências, denominadas humanísticas, são aquelas ligadas à capacidade de lidar com as inúmeras variáveis possíveis de se considerar quando as pessoas estão envolvidas. Essas competências trazem aspectos explorados pelas teorias de inteligências múltiplas, especialmente pelo campo de estudo da inteligência emocional. Estão relacionadas às questões humanas de relacionamento, de compreensão da realidade sob um prisma mais amplo, de comunicação, de liderança, de organização e de gestão, incluindo-se aí a gestão de si mesmo. As competências humanísticas elencadas na Resolução CNE/CES no 1/2003 estão relacionadas umas às outras, de forma interdependente. Pode-se concluir, ainda, que a menção às competências humanísticas não se relaciona meramente ao desenvolvimento de determinados comportamentos nos alunos, que podem vir a denotar a presença ou não da competência (19).

Espera-se da formação do Médico Veterinário que lhe proporcione o desenvolvimento das competências de maneira aplicada. Essa é uma condição essencial para a geração dos resultados requeridos pela sociedade, no que tange à Medicina Veterinária, e exigidos das Instituições de Ensino Superior (19).

O Curso de Bacharelado em Medicina Veterinária da PUCPR procura desenvolver no egresso, a formação generalista, humanista, crítica e reflexiva, apto a compreender e traduzir as necessidades de indivíduos, grupos sociais e comunidades, com relação às atividades inerentes ao exercício profissional, no âmbito de seus campos específicos de atuação em saúde animal e clínica veterinária; saneamento ambiental e medicina veterinária preventiva, saúde pública e inspeção e tecnologia de produtos de origem animal; zootecnia, produção e reprodução animal e ecologia e proteção ao meio ambiente. Também apresentar conhecimento dos fatos sociais, culturais e políticos da economia e da administração agropecuária e agroindustrial. Capacidade de raciocínio lógico, de observação, de interpretação e de análise de dados e informações, bem como dos conhecimentos essenciais de Medicina Veterinária, para identificação e resolução de problemas $(10,11,12,13)$.

A prática da multidisciplinaridade foi considerada ao elaborar as competências do Curso de Medicina Veterinária e cultivada na vida acadêmica, por meio de projetos, estágios e pelas atividades complementares desenvolvidas durante a permanência do estudante no Curso. Estas atividades são capazes de desenvolver no estudante uma visão geral do paciente, como a percepção da importância do envolvimento dos animais com as famílias e o seu significado para a saúde humana (13).

Dessa forma o egresso deste Curso de Medicina Veterinária deve ser reconhecido socialmente como um profissional qualificado e responsável, envolvido com as questões que regem a profissão, e também com o mundo globalizado (13).

\section{Grade curricular, competências desenvolvidas e estratégias de ensino}

Diante das diferenças sociais, econômicas e políticas entre países em relação às necessidades educacionais, a OIE propõe um Modelo de Currículo Núcleo em Medicina Veterinária (Quadro 2). Cabe a cada Estabelecimento de Ensino de Medicina Veterinária complementar o Modelo em conformidade com a sua realidade local e com suas necessidades educativas nacionais. No entanto, é preciso manter a intenção original do modelo - ou seja, educar estudantes de medicina veterinária para atingir as recomendações da OIE sobre as competências de se formar veterinários (23). 
Quadro 2. Modelo de Currículo Núcleo em Medicina Veterinária.

\begin{tabular}{|c|c|c|c|c|}
\hline \multirow[b]{2}{*}{$\begin{array}{c}\text { CONTEÚDO DO } \\
\text { CURSO }\end{array}$} & \multirow[b]{2}{*}{ 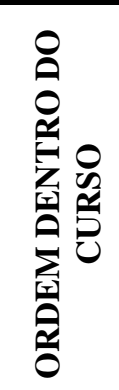 } & \multicolumn{3}{|c|}{ MÍNIMAS COMPETÊNCIAS ABORDADAS } \\
\hline & & 离易 & ESPECÍFICAS & AVANÇADAS \\
\hline Bioquímica & Início & $\begin{array}{c}\text { Todas } \\
* * *\end{array}$ & - & - \\
\hline Genética & Início & Todas & - & - \\
\hline Anatomia & Início & Todas & - & - \\
\hline Fisiologia & Início & Todas & - & - \\
\hline Imunologia & Início & Todas & - & - \\
\hline Biomatemática & Início & Todas & *Epidemiologia & - \\
\hline $\begin{array}{c}\text { Bem-estar animal e } \\
\text { Etologia }\end{array}$ & $\begin{array}{l}\text { Início e } \\
\text { meio }\end{array}$ & Todas & *Bem-estar animal & - \\
\hline Parasitologia & Meio & Todas & *Zoonoses & - \\
\hline $\begin{array}{l}\text { Farmacologia/ } \\
\text { Toxicologia }\end{array}$ & Meio & Todas & *Produtos veterinários & - \\
\hline Patologia & Meio & Todas & $\begin{array}{c}\text { *Epidemiologia } \\
\text { *Zoonoses } \\
\text { *Doenças de animais } \\
\text { transfronteiriças } \\
\text { *Doenças emergentes e re- } \\
\text { emergentes }\end{array}$ & - \\
\hline $\begin{array}{c}\text { Doenças } \\
\text { transmissíveis }\end{array}$ & Meio & - & $\begin{array}{c}\text { *Epidemiologia } \\
\text { *Zoonoses } \\
\text { *Doenças de animais } \\
\text { transfronteiriças } \\
\text { *Doenças emergentes e re- } \\
\text { emergentes } \\
\text { *Programas de prevenção e } \\
\text { controle de doenças } \\
\end{array}$ & $\begin{array}{c}\text { *Gestão de doenças } \\
\text { contagiosas }\end{array}$ \\
\hline Microbiologia & Meio & Todas & $\begin{array}{c}\text { *Zoonoses } \\
\text { *Doenças de animais } \\
\text { transfronteiriças } \\
\text { *Doenças emergentes e re- } \\
\text { emergentes } \\
\text { *Programas de prevenção e } \\
\text { controle de doenças }\end{array}$ & *Higiene de alimentos \\
\hline Epidemiologia & Meio & - & $\begin{array}{c}\text { *Epidemiologia } \\
\text { *Doenças emergentes e re- } \\
\text { emergentes } \\
\text { *Programas de prevenção e } \\
\text { controle de doenças } \\
\text { *Legislação veterinária e } \\
\text { ética }\end{array}$ & $\begin{array}{c}\text { *Gestão de doenças } \\
\text { contagiosas } \\
\text { *Higiene de alimentos } \\
\text { *Aplicação de análises de risco }\end{array}$ \\
\hline $\begin{array}{l}\text { Economia rural, } \\
\text { gestão de empresas } \\
\text { e produção animal }\end{array}$ & $\begin{array}{l}\text { Meio e } \\
\text { final }\end{array}$ & Todas & - & *Administração e gestão \\
\hline & & & & \\
\hline
\end{tabular}

Pimpão CT, Catapan DC, Rocha RMVM, Chi KD, Teixeira VN, Madeira HMF. et al. Desenvolvendo competências na aprendizagem de estudantes de medicina veterinária. Vet. e Zootec. 2017 Set.; 24(3): 592-603. 


\begin{tabular}{|c|c|c|c|c|}
\hline $\begin{array}{c}\text { Ciências clínicas e } \\
\text { de diagnóstico }\end{array}$ & $\begin{array}{l}\text { Meio e } \\
\text { final }\end{array}$ & - & *Produtos veterinários & - \\
\hline $\begin{array}{c}\text { Legislação } \\
\text { veterinária nacional } \\
\text { e internacional }\end{array}$ & $\begin{array}{l}\text { Meio e } \\
\text { final }\end{array}$ & - & $\begin{array}{c}\text { *Bem-estar animal } \\
\text { *Produtos veterinários } \\
\text { *Programas de prevenção e } \\
\text { controle de doenças } \\
\text { *Legislação veterinária e } \\
\text { ética } \\
\text { *Higiene de alimentos } \\
\text { *Procedimentos gerais de } \\
\text { certificação }\end{array}$ & $\begin{array}{c}\text { *Gestão de doenças } \\
\text { contagiosas } \\
\text { *Higiene de alimentos } \\
\text { *Organização dos serviços } \\
\text { veterinários } \\
\text { *Procedimentos de inspeção e } \\
\text { certificação } \\
\text { *Quadro do comércio } \\
\text { internacional }\end{array}$ \\
\hline $\begin{array}{l}\text { Nutrição e gestão } \\
\text { da saúde do } \\
\text { rebanho }\end{array}$ & Final & - & $\begin{array}{c}\text { *Epidemiologia } \\
\text { *Bem-estar animal } \\
\text { *Zoonoses } \\
\text { *Produtos veterinários } \\
\text { *Programas de prevenção e } \\
\text { controle de doenças } \\
\text { *Higiene de alimentos }\end{array}$ & $\begin{array}{c}\text { *Gestão de doenças } \\
\text { contagiosas } \\
\text { *Higiene de alimentos } \\
\text { *Aplicação de análises de risco } \\
\text { *Procedimentos de inspeção e } \\
\text { certificação }\end{array}$ \\
\hline Saúde pública & Final & - & $\begin{array}{c}\text { *Zoonoses } \\
\text { *Produtos veterinários } \\
\text { *Programas de prevenção e } \\
\text { controle de doenças } \\
\text { *Higiene de alimentos }\end{array}$ & $\begin{array}{c}\text { *Gestão de doenças } \\
\text { contagiosas } \\
\text { *Higiene de alimentos } \\
\text { *Organização dos serviços } \\
\text { veterinários } \\
\text { *Procedimentos de inspeção e } \\
\text { certificação } \\
\end{array}$ \\
\hline $\begin{array}{c}\text { Segurança e higiene } \\
\text { de alimentos }\end{array}$ & Final & - & $\begin{array}{c}\text { *Zoonoses } \\
\text { *Produtos veterinários } \\
\text { *Programas de prevenção e } \\
\text { controle de doenças } \\
\text { *Higiene de alimentos } \\
\text { *Legislação veterinária e } \\
\text { ética } \\
\text { *Procedimentos gerais de } \\
\text { certificação }\end{array}$ & $\begin{array}{c}\text { *Higiene de alimentos } \\
\text { *Procedimentos de inspeção e } \\
\text { certificação } \\
\text { *Quadro do comércio } \\
\text { internacional }\end{array}$ \\
\hline $\begin{array}{c}\text { Jurisprudência ética } \\
\text { e profissional }\end{array}$ & Final & - & $\begin{array}{l}\text { *Legislação veterinária e } \\
\text { ética } \\
\text { *Procedimentos gerais de } \\
\text { certificação }\end{array}$ & - \\
\hline Comunicação & Constante & Todas & *Habilidades de comunicação & *Administração e gestão \\
\hline
\end{tabular}

***Competências gerais / humanísticas (25): atenção à saúde, tomada de decisões, comunicação, liderança, administração e gerenciamento, educação permanente.

Fonte: (23).

A matriz curricular do Bacharelado em Medicina Veterinária da PUCPR foi baseada em competências que chamamos de basilares, que visam a formação integral do indivíduo, bem como sua atuação no contexto social, são elas: comunicar-se eficientemente nas formas escrita, oral, gráfica e artística; desenvolver raciocínio científico; atuar de forma ética e empreendedora; utilizar adequadamente as ferramentas tecnológicas; integrar os aspectos do desenvolvimento global de forma sustentável para a conservação da vida.

A partir das competências basilares foram trabalhadas as Competências Profissionalizantes, em que o estudante depara-se em cada disciplina com a aquisição de conhecimento, habilidade e atitude em uma área diferente da profissão, ou seja: realizar diagnóstico e terapêutica em medicina veterinária; realizar programas de medicina veterinária 
preventiva e de atenção geral à saúde pública; adquirir conhecimento para o manejo de rebanhos; atuar de forma ética na biotecnologia da reprodução e dos produtos biológicos e executar a inspeção sanitária e tecnológica de produtos de origem animal $(10,11,12,13)$.

Ao compararmos a matriz curricular do curso de Medicina Veterinária da PUCPR (13) com a matriz recomendada pela OIE (23), pode-se observar que a matriz desenvolvida na PUCPR está em concordância com a recomendação quanto a distribuição das disciplinas ao longo dos cinco anos de curso, nas disciplinas envolvidas no programa e no programa das disciplinas desenvolvidas em quase sua totalidade.

As divergências encontradas em relação a distribuição das disciplinas, se concentra somente na oferta da disciplina de microbiologia, pois a recomendação da OIE, é que esta disciplina se desenvolva na metade do curso e na matriz curricular aplicada na PUCPR, esta disciplina se concentra no inicio do segundo ano de curso (13).

Quanto as disciplinas ofertadas ou os programas desenvolvidos, a divergência percebida, seria na comunicação em outros idiomas, considerados oficiais pela OIE, ou seja, inglês, francês ou espanhol. Existe a previsão de ministrar disciplinas em inglês em nosso curso, porém isto deverá ocorrer a partir de 2016, porém a mesma disciplina ofertada em inglês, deverá ser ofertada em português (13).

O conteúdo a ser abordado no âmbito das disciplinas não deve sofrer alteração em virtude da adoção de novas estratégias de ensino-aprendizagem, somente atualizado. $\mathrm{O}$ mesmo conteúdo, trabalhado de uma maneira diferente pelo professor, pode produzir nos alunos resultados de aprendizagem diferentes (19).

Para que o professor do curso de medicina veterinária possa escolher e obter sucesso na implantação das estratégias de ensino-aprendizagem, o documento "Estratégias de ensinoaprendizagem para desenvolvimento das competências humanísticas", elaborado pelo Conselho Federal de Medicina Veterinária (19), exemplifica as diferentes estratégias de ensino e ao mesmo tempo, classifica quanto ao grau de preparo exigido do professor para sua implementação, conforme Quadro 3.

Quadro 3. Classificação das estratégias de ensino-aprendizagem do curso de medicina veterinária quanto ao grau de preparo exigido em termos de competências do professor para sua implementação.

\begin{tabular}{lc}
\multicolumn{1}{c}{ ESTRATÉGIAS DE ENSINO } & $\begin{array}{c}\text { GRAU DE PREPARO DO PROFESSOR } \\
\text { PARA UTILIZAÇÃO DE ESTRATÉGIA }\end{array}$ \\
\hline Aula expositiva dialogada & \\
Painel & \\
Portfólio & Nível de preparo mínimo \\
Seminário & \\
Solução de problemas & \\
Estudo de caso & \\
Estudo de texto & \\
Estudo dirigido & \\
Lista de discussão por meios informatizados & \\
Mapa conceitual & Nível de preparo médio \\
Dramatização & \\
Fórum & \\
Oficina de trabalho (ou workshop) & \\
Tempestade cerebral & \\
Tribunal do júri & \\
Jogos & \\
Aprendizagem Baseada em Problemas (Problem-Based & Nível de preparo alto \\
Learning - PBL) & \\
Aprendizagem baseada em projetos & \\
Estudo do meio & \\
Fonte: (19) &
\end{tabular}

Fonte: (19).

Pimpão CT, Catapan DC, Rocha RMVM, Chi KD, Teixeira VN, Madeira HMF. et al. Desenvolvendo competências na aprendizagem de estudantes de medicina veterinária. Vet. e Zootec. 2017 Set.; 24(3): $592-603$. 


\section{CONCLUSÕES}

Trabalhar o conceito de competência associado à estratégia e à aprendizagem é um desafio para os docentes e discentes universitários. Isso requer a adoção de estratégias de ensino inovadoras.

As competências, gerais ou humanísticas, específicas e avançadas, basilares ou complementares somadas às propostas de estratégias de ensino, se aplicadas com consistência e coerentemente com as demais etapas do processo de ensino-aprendizagem, como o planejamento e a avaliação, contribuirão para melhorar a qualidade do ensino do curso e perfil de estudantes de Medicina Veterinária.

A adoção da metodologia desenvolvida por competência deve ser realizada gradativamente ao longo do curso para que a qualidade do ensino não seja deturpada. Tanto docentes como discentes devem acostumar-se as novas abordagens do conhecimento. Principalmente porque os professores têm que receber um treinamento e também porque os estudantes brasileiros não têm o hábito de estudar o conteúdo que será abordado em sala de aula antes que ela ocorra. Além disso, a atual geração de estudantes tem pressa em ter a informação, porém não tem paciência em apreender.

Outro grande desafio na adoção da metodologia por competência é definir qual é a forma mais adequada e eficiente de avaliação das competências desenvolvidas pelos estudantes.

Atualmente a informação está a um clique com o uso da internet, porém o que percebemos é que essa informação para o nosso estudante é muito superficial e consequentemente não sedimenta em sua memória, portanto a adoção da metodologia desenvolvida por competência é uma ferramenta muito útil para superarmos essa informação superficial e capacitar o individuo com conhecimentos eficientes e permanentes para oferecer uma formação profissional adequada aos novos contextos mundiais.

\section{REFERÊNCIAS}

1. Decreto $\mathrm{n}^{\circ}$ 5.622, de 19 de Dezembro de 2005. Regulamenta o art. 80 da Lei $\mathrm{n}^{\circ} 9.394$, de Dezembro de 1996, que estabelece as diretrizes e bases da educação nacional. Diário Oficial da União. 20 Dez 2005.

2. Cotta RMM, Costa GD, Mendonça ET. Portfólios crítico-reflexivos: uma proposta pedagógica centrada nas competências cognitivas e metacognitivas. Interface (Botucatu). 2015;9(54):573-88.

3. Lei $n^{\circ}$ 9394, de 20 de Dezembro de 1996. Estabelece as diretrizes e bases da educação nacional. Diário Oficial da União. 23 Dez 1996.

4. Resolução CNE/CES 1, de 18 de Fevereiro de 2003. Institui diretrizes curriculares nacionais dos cursos de graduação em medicina veterinária. Diário Oficial da União. 20 Fev 2003.

5. Cotta RMM, Costa GD, Mendonça ET. Portfólio reflexivo: uma proposta de ensino e aprendizagem orientada por competências. Cienc Saude Coletiva. 2013;18(6):1847-56. 
6. Ferreira JL, Carpim L, Behrens MA. O professor universitário construindo conhecimentos inovadores para uma prática complexa, colaborativa e dialógica. Rev Dialogo Educ. 2013;13(38):69-84.

7. Aretio LG, Cordella MR, Blanco MG. Claves para la educación: actores, agentes y escenarios en la sociedad actual. Madrid: Narcea SA Ediciones; 2009.

8. Lima VV. Competência: distintas abordagens e implicações na formação de profissionais de saúde. Interface (Botucatu). 2005;9(17):369-79.

9. Masetto MT. Competência pedagógica do professor universitário. São Paulo: Summus Editorial; 2003.

10. Pontifícia Universidade Católica do Paraná. Resolução no 73/2012 - CONSUN, de 28 de Agosto de 2012. Projeto pedagógico institucional da PUCPR. Curitiba: PUCPR; 2012.

11. Pontifícia Universidade Católica do Paraná. Resolução n. ${ }^{\circ}$ 110/2013 - CONSUN, de 24 de Julho de 2013. Aprova reformulação do regimento geral da PUCPR [Internet]. Curitiba: PUCPR; 2013 [cited 2017 Feb 24]. Available from: http://www.pucpr.br/arquivosUpload/5370463411338897394.pdf

12. Pontifícia Universidade Católica do Paraná. Projeto pedagógico da escola de ciências agrárias e medicina veterinária. Curitiba: PUCPR; 2013.

13. Pontifícia Universidade Católica do Paraná. Projeto pedagógico do curso de medicina veterinária. Curitiba: PUCPR; 2013.

14. Gil AC. Métodos e técnicas de pesquisa social. 5a ed. São Paulo: Atlas; 1999.

15. Castro CM. Estrutura e apresentação de publicações científicas. São Paulo: McGraw-Hill; 1976.

16. Fonseca JJS. Metodologia da pesquisa científica. Fortaleza: Universidade Estadual do Ceará; 2002.

17. Ministério da Educação (BR). Referenciais para formação de professores. Brasília: MEC/SEF; 2002.

18. Fleury MTL, Fleury A. Construindo o conceito de competência. Rev Adm Contemp. 2001;5(n $\left.\mathrm{n}^{\mathrm{o}} \mathrm{esp}\right): 183-96$.

19. Conselho Federal de Medicina Veterinária. Estratégias de ensino-aprendizagem para desenvolvimento das competências humanísticas [Internet]. Brasília: CFMV; 2012 [cited 2015 Sept 2]. Available from: http://portal.cfmv.gov.br/uploads/files/Estrategias\%20de\%20Ensinoaprendizagem $\% 20$ para\%20Desenvolvimento $\% 20$ das $\% 20$ Competencias $\% 20$ Humanisticas _site.pdf 
20. Delors J. Educação: um tesouro a descobrir. Relatório para a Unesco da Comissão Internacional sobre Educação para o século XXI [Internet]. Brasília: Unesco; 2010 [acesso $2015 \quad$ Sept 2$]$. Available from: http://unesdoc.unesco.org/images/0010/001095/109590por.pdf

21. Mitre SM, Cotta RMM, Fortini IF, Raimundo IC, Souza NA, Santos RGC, et al. Flexibilidade, acesso e diversidade na formação universitária em saúde: o aprender fazendo no desenvolvimento de competências profissionais. Modelos flexibles de la organización de la formación. Rev CIDUI. 2014;1(2):1-15.

22. Cordeiro J. Didática. São Paulo: Contexto; 2007.

23. Organização Mundial de Saúde Animal, editor. Anais da 3a Conferência Mundial sobre Educação Veterinária e o Papel dos Órgãos Veterinários Estatutários; 4-6 Dez 2013; Foz do Iguaçu.

24. Organização Mundial de Saúde Animal. Recomendaciones de la OIE sobre las competências mínimas que se esperan de los veterinários recién licenciados para garantizar servicios veterinários nacionales de alta calidad. França: OIE; 2012.

25. Ministério da Educação (BR). Resolução CNE/CES 1, de 18 de Fevereiro de 2003. Institui diretrizes curriculares nacionais dos cursos de graduação em medicina veterinária [Internet]. Brasília; 2003 [cited 2015 Sept 2]. Available from: http://portal.mec.gov.br/cne/arquivos/pdf/ces012003.pdf

Recebido em: 20/05/2016

Aceito em: 09/08/2017 